\title{
L'expérience de l'étranger : vivre et enseigner en exil après le 2 décembre
}

\section{Sylvie Aprile}

\section{(2) OpenEdition}

\section{Journals}

Édition électronique

URL : https://journals.openedition.org/dhfles/1244

DOI : $10.4000 /$ dhfles. 1244

ISSN : 2221-4038

Éditeur

Société Internationale pour l'Histoire du Français Langue Étrangère ou Seconde

Édition imprimée

Date de publication : 1 juin 2004

ISSN : 0992-7654

Référence électronique

Sylvie Aprile, "L'expérience de l'étranger : vivre et enseigner en exil après le 2 décembre », Documents pour I'histoire du français langue étrangère ou seconde [En ligne], 32 | 2004, mis en ligne le 01 janvier 2012, consulté le 27 mai 2021. URL : http://journals.openedition.org/dhfles/1244 ; DOI : https:// doi.org/10.4000/dhfles.1244 




As a result of the imperial proscription against them, second Empire exiles are forced to reside abroad, sometimes for as long as twenty years. In order to survive, they have to learn the language of their new abode, England in most cases. often, they are also bound to be involved professionally in education. They need a job, and teaching French is among the opportunities offered to those who lack manual skills or are unable to work in their field. Most French people resent their teaching occupation which they see, or at least which they describe, as a compromise, even as a demeaning situation. Beyond such a discourse, we decided to research individual careers and teaching organisations, to analyse their ways and their role in setting up, in England and in France, a school system which grants a new status to the teaching of modern

Même s'il ne peut être vécu que comme provisoire voire éphémère, l'exil politique qui suit le coup d'État de 1851 conduit les proscrits français à une installation durable dans des pays non francophones: Angleterre, Belgique flamande ou États-Unis. Pour vivre, l'une des principales activités qui s'offre à eux est l'enseignement du français. Il faut alors tout à la fois apprendre la langue de l'autre et enseigner la sienne. Ce milieu de la proscription en Grande-Bretagne contribue, comme ailleurs en Europe, à la structuration au cours des années 1860-1880 d'une réflexion et d'une organisation de la profession de professeur de français à l'étranger.

Ces exilés posent également un certain nombre de questions connexes : celle de l'éducation du peuple et celle du rôle de la langue dans le contexte de la Revanche. Ce qui est en jeu n'est pas seulement une démarche pédagogique mais l'image en 
construction de la France républicaine et patriotique. En dehors de la simple découverte d'aspects méconnus des transferts, exportations et médiations culturels qui s'opèrent par le biais de l'exil, ce sont à la fois les pratiques de l'enseignement au quotidien et leurs constantes mises en perspective avec une actualité ou un devenir politique qui seront présentées ici.

Si les travaux de Paul Gerbod, auteur de plusieurs réflexions sur l'enseignement de la langue française en Grande-Bretagne au XIX siècle $^{1}$ apparaissent pionniers, ceux sur les transferts culturels de Michel Espagne, ou ceux de sociologues ont permis de repenser la place de l'exil dans une perspective d'histoire sociale tout autant que politique. On pose généralement en termes de créativité et donc de culture la question de l'exil, peu en termes d'emplois, d'apprentissages ou d'imitations ; il faut donc payer sa dette aux analyses sur l'immigré d'Abdelmalek Sayad. J'ai pu enfin confronter mes recherches tout récemment aux travaux d'Adèle Thomas, jeune doctorante qui a analysé à travers la Société française des professeurs de français cette volonté de diffusion du français qui se renforce à la fin du XIX ${ }^{\mathrm{e}}$ siècle $^{2}$.

Il s'agit ici de comprendre comment se construit cette communauté française, comment en s'inscrivant dans la durée, elle investit un certain nombre de positions et de pratiques qui aboutissent à la création d'instances chargées en fin de siècle de protéger les enseignants et d'améliorer l'apprentissage de la langue française.

\section{La formation d'une communauté enseignante}

Près d'un millier de proscrits s'installent en Grande-Bretagne. Ce choix est le plus souvent contraint. Ils souhaitent s'installer aux frontières de la France en pays francophones, mais ils sont soumis en Suisse et surtout en Belgique à une législation sur les étrangers qui rend leur statut précaire et leur présence peu désirée. A ce souci de préserver de bonnes relations diplomatiques avec la France, s'ajoute surtout en Belgique la crainte d'une concurrence dans les professions libérales et l'enseignement. Cependant les «intellectuels français » sont accueillis à bras ouverts dans les universités suisses et en Amérique latine. La présence des exilés est également temporaire : ils sont nombreux à rentrer à la faveur des amnisties en 1853 et surtout en 1859, mais ils vont pour certains s'enraciner notamment en Angleterre pour la durée totale de l'Empire, soit près de 20 ans, période qui est aussi celle de leur maturité, donc de leur activité professionnelle. Cette installation est parfois même définitive puisque certains d'entre eux sont encore très actifs dans les années 1880. Ce sont dans certains cas aussi leurs fils qui leur succèdent comme enseignants de français en Angleterre.

Sur qui et sur quelles pratiques repose cet enseignement à la mi-XIX ${ }^{\mathrm{e}}$ siècle ? En d'autres termes dans quel paysage prennent-ils place ?

L'enseignement du français repose déjà traditionnellement sur la présence de réfugiés français. Il existe en effet une tradition de l'enseignement par les réfugiés. Après la révocation de l'Édit de Nantes en 1685, cet enseignement est dominé par

\footnotetext{
${ }^{1}$ Paul Gerbod, «L'enseignement de la langue française en Grande-Bretagne au XIX ${ }^{\text {ème }}$ siècle », Documents pour l'histoire du français langue étrangère ou seconde, $\mathrm{n}^{\circ} 2$, décembre 1988 et «L'enseignement de la langue française en Angleterre au XIX ${ }^{\mathrm{e}}$ siècle (1800-1860) », L'Information historique, vol. 53, n 5,1991 , p. 180-186.

${ }^{2}$ Adèle Thomas, «L'enseignement du français en Grande-Bretagne : analyse et (ré)action des Français de la Société nationale des professeurs de français en Angleterre dans les années 1880 », DEA Paris Nord, Relations interculturelles anglophones et françaises, sous la direction de Mme Rose May Pham Dinh, septembre 2002. La quatrième partie de cet article s'appuie pour une large part sur ses recherches. Sur ce thème voir également Albert Salon, «La diffusion du français hors des pays francophones et francisants », in Gérald Antoine et Robert Martin (dir.), Histoire de la langue française 1880-1914, Paris : Éditions du CNRS, 1985, p. 421.
} 
l'influence des huguenots. La Révolution française a ensuite conduit certains aristocrates, exilés en Angleterre, à s'improviser maîtres de langue, tandis que des prêtres émigrés créaient des écoles où ils enseignaient le français. Puis, c'est l'instabilité de régime politique que connaît la France au XIX ${ }^{\mathrm{e}}$ siècle, qui entraîne un nouveau flot d'émigration continue de Français vers l'Angleterre, qui à leur tour enseignent le français. Comme l'a résumé Paul Gerbod :

On recense successivement les bonapartistes chassés par la Terreur Blanche en 1815, les ultras de 1830, les orléanistes de 1848, les « démocrates » de 1848 et les opposants au coup d'État du 2 décembre 1851. En 1871, ce sont les «Communards » échappés à la répression des Versaillais et de Monsieur Thiers. Parmi eux, un certain nombre pour des raisons alimentaires «courent le cachet ». D'origine bourgeoise, bacheliers ès lettres ou licenciés en droit, ils sont en mesure de se transformer en maîtres de français ${ }^{3}$.

Les données quantitatives sont difficiles à établir, surtout pour les exilés du Second Empire, car les recensements britanniques ne sont pas d'un grand secours. Ils ont lieu en 1851 soit avant leur arrivée, puis en 1861 date à laquelle bon nombre sont repartis. Mais ce qui est certain, c'est que ces vagues d'exilés se traduisent à chaque fois par une pression sur le marché de l'enseignement ${ }^{1}$. Le communard Vermersch décrit ainsi dans une lettre à son ami Vuillaume datée du 10 décembre 1872 le déséquilibre entre l'offre et la demande de professeurs de français :

c'est à peu près l'habitude: on ne paie pas les maîtres de français, et cependant on en trouve autant qu'on veut ; ce n'est pas étonnant, il y a tous les dix ou quinze ans maintenant des émigrations qui donnent à l'Angleterre une quantité de Parisiens [...]. Beaucoup n'ont pas d'état manuel : ils donnent des leçons de leur langue, d'où abondance de professeurs, et, comme disent les économistes, encombrement du marché et baisse des salaires ${ }^{4}$.

Si on ne peut guère compter les exilés, on peut les localiser. Ils sont surtout installés à Londres et ses environs. A Londres, ils s'installent surtout à Soho, quartier français qui a mauvaise réputation auprès des Français eux-mêmes qui cherchent à s'en éloigner, ou au moins à s'installer sur ses marges. On peut à ce titre citer les témoignages de Jules Vallès, Élisée Reclus, Alphonse Esquiros. On peut ainsi reconstituer aisément les topoi qui accompagnent côté anglais et français cet enseignement. Jusqu'au milieu du XIX siècle, l'enseignement du français en Angleterre se déroule essentiellement dans le cadre du préceptorat particulier ou bien encore au sein d'une académie privée de langues. Pour autant, s'ils ont su s'imposer dans ce domaine en Angleterre, les Français doivent faire face à un certain nombre de critiques. Les Anglais redoutent en effet que les Français ne corrompent leurs élèves même s'ils craignent moins qu'auparavant que les enseignants catholiques ne fassent preuve d'un certain prosélytisme auprès des enfants dont on leur a confié l'éducation.

Les perceptions et représentations de l'enseignant français sont construites sur les stéréotypes qui caractérisent tout Français: celui-ci est léger et peu compétent. L'enseignant français doit faire face à l'attitude irrespectueuse de ses élèves, liée à son incompétence à se plier et exercer toute discipline. Paradoxalement, il semble que, dans le même temps, la présence d'un Français dans une école ait permis d'en rehausser le prestige. Cette situation semble évoluer à partir de 1850. En effet, à l'occasion de l'exposition universelle de 1851 à Londres, l'Angleterre découvre l'industrialisation croissante de pays comme la Prusse, la France ou les États-Unis, s'en inquiète, et tente d'en comprendre les raisons. La qualité comparée de l'enseignement secondaire et

\footnotetext{
${ }^{3}$ Paul Gerbod, «L'enseignement de la langue française en Angleterre au XIX ${ }^{\mathrm{e}}$ siècle (1800-1860) », op. cit., p. 181.

${ }^{4}$ Marc Vuillaume, Mes cahiers rouges au temps de la Commune, Paris, Albin Michel, 1971, p. 43.
} 
technique devient un élément du débat sur la puissance industrielle et commerciale des pays. Ceci s'accroît encore dans les années 1860: l'on attribue en partie à une supériorité atteinte par la Prusse en matière éducative, ses victoires face à l'Autriche, en 1866, puis à la France, en 1870. Les hommes politiques et industriels britanniques s'attachent, du moins dans leur discours, à porter une plus grande attention au système d'enseignement secondaire à destination d'un plus large public. Les proscrits français débarquent donc en Grande-Bretagne dans le cadre d'une tradition et d'interrogations nouvelles sur l'enseignement.

\section{Une inscription dans la durée}

En général, être maître de langue est une profession par défaut et provisoire qui ne nous est connue que par les récits autobiographiques et les échanges épistolaires. Les informations données sont souvent périphériques voire anecdotiques et s'inscrivent dans l'expression d'une anglophobie qui a des visées communautaires ${ }^{5}$. A contrario, la correspondance révèle le rôle central de cet enseignement, souvent la seule source de revenus réguliers. Il faut alors bénéficier de réseaux de recommandation qui passent parfois par la France : Pierre-Alfred Talandier sollicite une lettre de recommandation de Jules Michelet pour un poste dans une école militaire.

Certains exilés français connaissent semble-t-il déjà l'anglais. Victor Schœlcher a une connaissance sommaire mais réelle de la langue, qu'il est à son arrivée capable d'écrire et de lire ${ }^{6}$. S'agit-il des souvenirs d'un enseignement qui lui a été dispensé ou plus certainement d'un usage de cette langue dans ses voyages et séjours outreatlantique?

Rappelons que les proscrits ne sont pas des jeunes gens et que leurs années d'études sont souvent loin derrière eux. L'apprentissage de la langue anglaise se fait donc essentiellement sur le «tas », souvent par une pratique écrite de la version et du thème à l'imitation de l'enseignement des langues mortes plus que par un enseignement oral. On possède peu de témoignages de cette pratique. Alexandre Ledru-Rollin travaille pour sa part le thème et la version. Ses archives contiennent les exercices anglo-français où sur trois cahiers d'avril à décembre 1857, il a appris la langue anglaise. Il s'agit de traductions par thème d'exercice : études des propositions, verbes les plus usités et verbes irréguliers. L'absence de ratures pose la question d'une simple transcription ou d'un travail exemplaire ${ }^{7}$. Cet apprentissage paraît d'ailleurs tardif à double titre : LedruRollin est arrivé en Angleterre en 1849 soit huit ans plutôt, il est d'autre part déjà marié à une Anglaise. Le mariage mixte ou le concubinage ne paraissent guère avoir ici d'incidence, ils sont pourtant courants en exil: Alphonse Esquiros a également une compagne anglaise ${ }^{8}$. Cela le conduit a contrario à une immersion dans la langue

\footnotetext{
${ }^{5}$ Sylvie Aprile, «Translations politiques et culturelles: les proscrits français et l'Angleterre », Genèses, mars 2000, p. 35-56.

${ }^{6}$ La correspondance de Victor Scholcher, présentée par Nelly Schmidt, Paris, Maisonneuve et Larose, 1995.

${ }^{7}$ B.H.V.P., Papiers Ledru-Rollin, MS2028, études de langues. On y trouve également un texte en anglais sur la musique, les phonéties, des exercices aussi en allemand. MS2025, achats de dictionnaires français-anglais.

${ }^{8}$ Alphonse Esquiros (1812-1876). Poète, collaborateur de La France littéraire, est l'auteur avant l'exil de plusieurs ouvrages qui l'ont rendu célèbre : l'Évangile du Peuple, les Vierges folles (contre l'ignominie de la prostitution) et les Vierges martyres écrit avec son épouse Adèle qui lui inspire en grande partie cet ouvrage «féministe ». Durant son exil, il vit en Belgique et surtout en Angleterre où il devient le collaborateur de la Revue des deux mondes. Il se sépare d'Adèle pour vivre avec une Anglaise qui lui donne un fils William. Les ouvrages qu'il a publiés sur l'Angleterre et l'histoire de l'Angleterre sont fort nombreux: La morale universelle, les moralistes anglais (1859). Itinéraire descriptif et
} 
anglaise. Les lettres d'Esquiros témoignent de glissements sémantiques, d'anglicismes qui accompagnent son discours sur la langue. En 1856, il écrit à Victor Hugo, alors même qu'il fait des chroniques pour la Revue des deux mondes:

Je ne lis que les journaux anglais, je ne parle qu'anglais. C'est vous dire si je parle mal ! Je suis cherchant ici des sujets d'étude sur l'Angleterre et du travail ${ }^{9}$.

Tout autre mais plus détaillé encore est le témoignage de Martin Nadaud, ancien maçon et ex-représentant du peuple. Dans son autobiographie, Léonard, maçon de la Creuse, il raconte son étude de la langue et de la culture anglaises, faite à la fois par la fréquentation des livres et des hommes ${ }^{10}$. Il raconte l'accueil chaleureux qu'il a reçu de « Jean » Ludlow, l'un des principaux membres du mouvement chartiste :

Mon ami m'encouragea à apprendre la langue de son pays. Il avait même entrepris de me donner des leçons de latin, mais il ne tarda pas à s'apercevoir que j'étais un fort mauvais élève ${ }^{11}$.

Martin Nadaud accepte de travailler sous les directives d'un proscrit, Pierre Barrère, qui tenait une pension à la Châtre avant la seconde république. Pendant deux mois, il apprend l'anglais et se perfectionne en grammaire française. Barrère le met ensuite en relation avec un placier proche de Trafalgar square, il trouve alors un poste à Brighton, puis à Putnay. Puis il reprend le poste occupé par un autre proscrit, Tristan Duché, à Wimbledon.

Le passage de l'apprentissage de l'anglais à l'enseignement du français se fait souvent de façon très rapide. Le cas de Nadaud est là encore très spécifique car il devient enseignant parce qu'il ne trouve pas de travail comme maçon. L'enseignement est perçu comme un déclassement, un travail de domestique qui n'est guère compatible avec la liberté d'esprit des républicains ou socialistes. Pour survivre, tous les exilés n'ont pas de talent de plume ni forcément de relations éditoriales privilégiées. Il faut tout simplement travailler, et l'enseignement du français est l'un des seuls emplois possibles pour des hommes qui n'ont pas de qualification manuelle ou qui ne peuvent exercer leur activité professionnelle d'avocat ou de journaliste. C'est à leurs yeux, entrer dans la livrée des précepteurs soumis, c'est faire «ce métier de chien » selon l'expression que l'on trouve si souvent sous l'Ancien Régime sous la plume des précepteurs français. Parmi tant d'autres proscrits, Gustave Lefrançais et Pierre Leroux témoignent de cette représentation négative. Ils parlent avec un certain mépris de leurs

historique de la Grande-Bretagne et de l'Irlande (1865). Religious life in England (1867). L'Angleterre et la vie anglaise (1869), traduction de l'ouvrage de Oliver Goldsmith, Voyage d'un chinois en Angleterre (1860)

Le fils d'Esquiros est diplômé en 1869 d'un BA de français délivré par l'université de Londres. Il revient en France avec son père en 1870 pour lui servir de secrétaire. Il meurt cette même année de la fièvre typhoïde. Ces enfants issus d'un métissage culturel n'ont pas non plus fait encore l'objet de recherches approfondies pour le $\mathrm{XIX}^{\mathrm{e}}$ siècle.

${ }^{9}$ A. Zielonska, Alphonse Esquiros, choix de lettres, Champion/Slatkine.

${ }^{10}$ Martin Nadaud (1815-1898). Ouvrier maçon creusois, élu représentant de son département à l'assemblée législative en 1849, est exilé en Grande-Bretagne après le coup d'État. Il reprend tout d'abord son métier de maçon puis devient professeur de français et d'histoire à l'école militaire préparatoire de Wimbledon. Il a publié sur l'Angleterre et surtout les ouvriers anglais plusieurs ouvrages : Histoire des classes ouvrières en Angleterre (1872), Questions ouvrières en Angleterre et en France (1884). Dans la préface de cet ouvrage, il évoque ses lectures : «Je lus les meilleurs historiens de l'Angleterre, Hallam, Kemble, Forster pour la période moderne, Macaulay, Buckle, Froude pour la période moderne. Je fis des recherches au British Museum, dans les revues, dans le Parliament Debat qui est un moniteur universel de nos voisins et j'arrivai à me convaincre que les faits énoncés par M. Ludlow, étaient confirmés par l'histoire. » Il mène d'autres études grâce à des ouvrages d'économie politique, ceux de sir Frédéric Eden, John Wade.

${ }^{11}$ M. Nadaud, Léonard, maçon de la Creuse. Maspero, 1977, p. 187. 
élèves, bourgeois anglais satisfaits d'eux-mêmes ${ }^{12}$. Tous deux s'en échappent. Les informations qui nous sont données portent surtout sur la perte d'indépendance qui accompagne cette profession. Les proscrits «barbus » engendrent la méfiance et sont concurrencés par les domestiques français qui quittent leur livrée pour enseigner. Gustave Lefrançais nous livre cependant quelques bribes de sa manière d'enseigner. Dans les souvenirs d'un révolutionnaire, il raconte qu'il doit inculquer le français à un surgeon chemist (pharmacien). Il est embarrassé car il ne connaît pas un mot d'anglais, et son élève pas un mot de français. Il trouve une solution :

J'ai alors un trait de génie, j'invente à notre usage respectif la méthode intuitive. J'apprends successivement à mon élève et en les lui faisant répéter jusqu'à ce qu'il les prononce à peu près bien les noms de tous les objets, viennent ensuite les mots se rapportant à leur forme, à leur composition, leur couleur, leurs dimensions, leurs usages, nous passons ensuite à l'action avec toutes ses particularités de temps et de personnes ${ }^{13}$.

C'est une réussite, car l'Anglais est en mesure de faire son voyage en France, mais Lefrançais perd alors sa source de revenus.

La situation n'est supportable que si l'on quitte le préceptorat pour un enseignement plus institutionnalisé, moins mercenaire dans des institutions et surtout dans les écoles de préparation militaire qui vont se peupler d'exilés français. Le cas n'est pas seulement anglais, Madier de Montjau enseigne en école militaire en Belgique. Une autre ressource existe, les mechanic's schools, où l'enseignement du français est aussi pratiqué.

Martin Nadaud devient maître de pension puis enseignant dans une école de préparation à l'entrée au collège militaire de Woolwich. Il faut noter que si cette promotion est beaucoup moins spectaculaire pour la plupart des proscrits, l'enseignement dans les écoles militaires est une fonction lucrative et somme toute prestigieuse. Elle semble réservée à une élite qui pratique un métissage culturel intense. Outre Nadaud, il faut citer Joseph Savoye, Théodore Karcher, Pierre-Alfred Talandier et Alphonse Esquiros qui sont des examinateurs réputés ${ }^{14}$. Les écoles militaires et les écoles de préparation militaire font-elles sciemment ou à leur insu appel à ces proscrits français? On l'ignore, la lettre de recommandation de Michelet que présente l'un d'entre eux a une valeur plus littéraire certainement que politique. Tous ces témoignages et exemples ne semblent guère marqués par une réflexion sur la fonction et la pratique enseignantes, or il est certain que nous sommes alors dans une période de transformation à laquelle, à leur corps défendant parfois, les proscrits vont participer.

\section{Les voies de la professionnalisation}

Jusqu'au milieu du $\mathrm{XIX}^{\mathrm{e}}$ siècle, l'apprentissage du français est laissé à la libre initiative personnelle. A partir de 1850 on voit l'enseignement des langues vivantes se développer hors du cadre privé. Ce développement est favorable aux enseignants de français, mais le français ne monopolise pas la place dévolue aux cours de langues. L'allemand participe lui aussi de ce combat pour la reconnaissance des langues vivantes face au prestige des langues classiques et entre en concurrence avec la langue des proscrits. Le français ne distance l'allemand que plus tard, en raison notamment de

\footnotetext{
${ }^{12}$ A. Zévaes, «Les proscrits français à Londres », Bulletin de la société d'histoire de la Révolution de 1848 et des révolutions du XIX ème siècle. Voir Pierre Leroux, La grève de Samarez, op. cit. et Gustave Lefrançais, Souvenirs d'un révolutionnaire, Futur antérieur, 1972.

${ }^{13}$ G. Lefrançais, op. cit., p. 170.

${ }^{14}$ Pierre-Alfred Talandier, (1822-1890), avocat et journaliste. A son retour, il est chargé de cours d'anglais au collège Henri IV. Il est révoqué de son poste, après son élection comme conseiller municipal en 1874. Il est ensuite député de Sceaux de 1876 à 1885.
} 
circonstances politiques après la Première Guerre mondiale. Ce nouveau paysage va de pair chez les proscrits avec une professionnalisation liée à leur enracinement. Ceci peut être envisagé à partir de parcours individuels et familiaux. Prenons tout d'abord le cas de la famille Barrère, qui produit deux générations d'enseignants, avec des aller-retours significatifs.

Le père, Pierre, répétiteur au lycée Bonaparte qui exerçait à la Charité-sur-Loire avant 1848, est venu s'installer à Londres en 1853 avec sa famille. Les premières années sont difficiles, mais en 1858 il est professeur de français à Wimbledon puis il obtient une chaire de professeur de français à l'Académie militaire avant de devenir lecturer pour les officiers de l'institut royal d'artillerie. Il est aussi l'auteur d'une étude plusieurs fois rééditée : Les écrivains français, leur vie et leurs æuvres ${ }^{15}$.

Il est de retour en France après 10 ans, mais n'abandonne pas ses relations avec l'Angleterre, puisqu'il dirige à Chatou un collège international. Ces collèges internationaux sont semble-t-il conçus comme l'équivalent pédagogique du libreéchange. L'idée en a été lancée lors de l'exposition universelle de 1862 par des exilés français et matérialisée par la fondation par Cobden de la Société d'éducation internationale administrée côté français par Michel Chevalier. Le principe est de dispenser un enseignement dans une langue étrangère au cours d'une année préparatoire puis de poursuivre ses études dans un établissement étranger affilié. L'initiative en revient au collège de Spring Grove, près de Richemond, dont le collège international de Chatou devient le partenaire français ${ }^{16}$. Pierre Barrère qui meurt en 1868 , ne le dirige que quelques années.

Son fils, Albert, enseigne l'anglais à Chatou jusqu'en 1870. Impliqué dans la Commune, il revient en Angleterre, professeur à la Merchant Taylor School, puis en 1877, à Cheltenham Collège. En 1879, sur les traces de son père, il enseigne à Woolwich et à l'institut royal d'artillerie, et publie des dictionnaires bilingues de vocabulaire militaire et argotique ${ }^{17}$.

Il n'est guère étonnant de retrouver les noms d'autres exilés et fils d'exilés parmi les auteurs de manuels d'enseignement. C. A. Chardenal, enseignant à Glasgow chez qui Nadaud a séjourné, a publié First french course or rules and exercises, London, chez W. Collins, s.d., Charles Cassal et Theodore Karcher sont les auteurs de The graduated course of translation from english into french, part II, the senior course with vocabulary of idioms and difficulties chez Longmans. Ces ouvrages sont destinés à des élèves et à des enseignants et marqués par la volonté d'être pratiques. Au-delà de l'argument de vente, il s'agit de s'inscrire dans un système économique et éducatif moderne.

La professionnalisation se marque aussi par la promotion en France du système scolaire britannique, avec d'autres pratiques comme la traduction ou l'essai. Ainsi Martin Nadaud montre-t-il dans l'ouvrage qu'il publie à son retour en France, l'Histoire des classes laborieuses en Angleterre, l'importance du système scolaire fondé par l'ouvrier Joseph Lancastre, un des pionniers de l'enseignement mutuel. Nadaud y parle également du système britannique de l'enseignement professionnel.

Alfred Esquiros a lui aussi été fortement marqué par le système d'enseignement britannique et l'un de ses ouvrages intitulé L'Émile $d u$ XIX ${ }^{\text {ème }}$ siècle en porte la marque. Cet ouvrage qui se veut un prolongement de l'ouvrage de Rousseau est un dialogue

\footnotetext{
${ }^{15}$ Pierre Barrère, Les écrivains français, leur vie et leurs auvres ou l'histoire de la littérature française, Paris, E. Lalouette, $2^{\mathrm{e}}$ édition, 1881.

${ }^{16}$ Impartial de la Nièvre, 02/08/67.

${ }^{17}$ A. Barrère, Argot and slang, a new french and english dictionnary of the cantwords, London, C. Whittingham. 1887. A. Barrère, A Dictionnary of english and french military terms, London Hachette, 1895-1896.
} 
entre un proscrit politique qui s'engage sur un navire et son épouse concernant l'éducation de leur enfant. Le père conseille qu'elle l'élève en Angleterre, pour bénéficier d'un climat plus vif et d'une plus grande liberté d'éducation. Les républicains rejoignent ici l'appréciation des libéraux sur le système scolaire anglais ${ }^{18}$.

Pierre-Alfred Talandier est pour sa part en 1861 l'auteur de L'Histoire des Pionniers équitables de Rochdale, et donc l'un de ceux qui font connaître le mouvement coopératif anglais en France. Son ouvrage qui ne peut être publié in extenso en France paraît en feuilleton dans le journal Le Progrès de Lyon d'octobre 1862 à avril 1863. Son influence est attestée par la formation quelque temps plus tard, à Lyon, de la première société coopérative française de type rochdalien : la Société des Travailleurs réunis de Perrache-Oullins. Alfred Talandier fournit également de la documentation au Crédit au travail dont il est l'un des souscripteurs. Il écrit aussi dans le journal l'Association, publiant notamment un article sur un magasin de gros fonctionnant à Manchester ${ }^{19}$. Il traduit aussi un des best-sellers de l'édition d'ouvrages à destination des classes populaires, le Self Help de Smiles.

C'est donc à nouveau en marge des échanges politiques que sont les transferts les plus féconds. Cette communauté repose sur une certaine proximité politique. Ce sont pour la plupart, semble-t-il, des proches de Louis Blanc qui donne lui même des conférences en anglais. Attitude fort éloignée de celle d'un Ledru-Rollin, auteur d'un ouvrage qui fait scandale à sa parution en 1849 : de la décadence de l'Angleterre.

Une dernière analyse à partir d'une autre trajectoire individuelle montre la nécessité de croiser les sources. C'est le cas de Alfred Hamonet, personnage central pour cette étude. Alfred Hamonet apparaît essentiellement dans les sources politiques de l'exil comme un exilé modeste et dont la vie a été brisée par le coup d'État. Il était sous la seconde république clerc de notaire, condamné à la déportation en Algérie, il a finalement réussi à s'installer à Londres, où il demeure encore en 1905, s'intitulant luimême le doyen des exilés. Dans le cadre de la loi de réparation nationale de 1881, qui indemnise les victimes du coup d'État, il est en effet pensionné du 2 décembre, mais le montant octroyé de $600 \mathrm{~F}$ lui paraît insuffisant et il adresse une réclamation à la commission départementale qui lui avait proposé au préalable la somme de $1200 \mathrm{~F}$ :

En témoignage apparemment de ma vive résistance au crime de décembre et en réparation du tort considérable qu'il m'avait causé. Serait-ce parce que je suis condamné par la force des intérêts à rester en exil. Serait-ce parce que je n'ai jamais sollicité un emploi du gouvernement. Serait-ce parce qu'à l'étranger je n'ai cessé de rendre service à mon propre pays par mes publications et diverses fonctions publiques?

Son dossier est complété par des attestations qui montrent qu'Hamonet était à son départ sur le point d'acquérir une étude; que sa vie a été brisée, que sa situation est précaire en Grande-Bretagne, avec 4 enfants en bas âge.

Dans Épisodes du coup d'État en 1851 dans la Sarthe. Une évasion, troisième et dernière partie de l'étude historique sur Charles Granger et son époque par Léon Guyon, avec une préface d'Alfred Hamonet qui est le récit de la vie d'un autre proscrit, il se met en scène dans la préface. Hamonet indique qu'il a demandé un poste en 1892 dans les perceptions françaises, demande restée sans réponse. Il conclut en déclarant : «faudra-til laisser mes os à l'étranger?»

\footnotetext{
${ }^{18}$ Cf. H. Taine, Notes sur l'Angleterre, 1871 et E. Marguerin et A. Mothoré, de l'enseignement des classes moyennes et des classes ouvrières en Grande-Bretagne, rapport à monsieur le sénateur, préfet de la Seine, imp. Charles de Mourgues frères, 1864, 272 p.

${ }^{19}$ Antoine Perrier, «Pierre-Alfred Talandier (1822-1890)», Bulletin de la Société archéologique et historique du Limousin, tome 100, 1973, p. 229-240.
} 
D'autres sources donnent une image quelque peu différente du personnage et explicitent ces services rendus, qui font de lui un exilé qui a réussi. Hamonet, publie à Londres dans les années 1860 un guide, édité par les éditions Hachette qui s'intitule l'Annuaire commercial et industriel ou guide général à l'usage des Français en Angleterre $^{20}$. Il est alors secrétaire de la société française de bienfaisance fondée sous la Monarchie de Juillet, qui bénéficie sous l'Empire de la bienveillance du consul général, et qui montre à la fois son insertion et même son rôle éminent dans l'organisation de la vie de la colonie française en Grande-Bretagne. Cet ouvrage, publié chaque année, dresse la liste par profession de tous les Français vivant en Grande-Bretagne, donne le nom des associations professionnelles et délivre des conseils de placement. Il y fait une large place à la pratique enseignante et fournit la liste des 48 professeurs qui appartiennent alors à l'association des professeurs de langue française. Ces annuaires sont publiés pendant au moins une vingtaine d'années.

Cela montre les effets de sources, et se situer à la marge change l'éclairage et la lecture. Alfred Hamonet ne «ment» pas, mais présente seulement, selon les publics auxquels il s'adresse, une posture distincte. Ces présentations de soi s'inscrivent dans une socio-histoire complexe de l'exil ${ }^{21}$.

\section{L'institutionnalisation d'un milieu}

Ce rôle des exilés aurait pu se limiter à la période de la proscription ou du moins on aurait pu concevoir que leur influence décline après la chute de l'Empire. Or au contraire les années 1880 sont marquées par l'essor de la Société nationale des professeurs de français en Angleterre (SNPFA), fondée à Londres le 12 novembre 1881 et qui tient son premier congrès en 1882, suivi de réunions annuelles. S'y ajoute un journal le Français. Les sociétaires fondateurs se caractérisent par leur engagement durable dans la vie politique et associative, avant et après leur exil. On y retrouve notamment Charles Cassal présenté dans le compte rendu du congrès de 1885 comme un ancien représentant du Peuple et surtout y apparaît la figure centrale d'Alfred Hamonet ${ }^{22}$. Gustave Rudler qui retrace l'histoire de la société dans les années 30 fait état du fait qu'Alfred Hamonet est « un homme de $48 »^{23}$. Il souligne par ailleurs, qu'au sein de la SNPFA «l'élément communard a dû être assez nombreux, cette nouvelle vague d'exil renforçant en quelque sorte la précédente ». Les autres personnalités sont pour la plupart déjà mentionnées comme enseignants dans le guide Hamonet des années 1860 et ont aussi d'autres fonctions dans la notabilité française. Georges Petilleau est le premier président de la section scientifique et littéraire de la Société nationale française $^{24}$. Il est aussi représentant de la Société des Gens de Lettres en Angleterre depuis les années $1870^{25}$. Parmi les 66 membres honoraires que compte la SNPFA en 1885,41 personnes sont également membres de la Société nationale française ${ }^{26}$.

\footnotetext{
${ }^{20}$ A. Hamonet, Annuaire commercial et industriel ou guide général à l'usage des Français en Angleterre, à Londres chez l'auteur, 32 Dockwille street, Picadilly W. A Paris chez Hachette et cie, 14 rue Pierre Sarrazin.

${ }^{21}$ On en trouvera une illustration méthodologique particulièrement pertinente dans Pierre Swiggers, «Histoire et historiographie de l'enseignement du français : modèles, objets et analyses », Études de Linguistique Appliquée, ${ }^{\circ}$ 78, avril-juin 1990, p. 31.

${ }^{22}$ Ibid., p. 35.

${ }^{23}$ SNPFA, La Journée du Cinquantenaire de la Société des Professeurs de français en Angleterre, tenue sous la présidence de Son Excellence, M. de Fleuriau, Ambassadeur de France en GrandeBretagne, le samedi 31 octobre 1931, 76 p., p. 12.

${ }^{24}$ Ibid., p. 7.

${ }^{25}$ Ibid., p. 11.

${ }^{26}$ SNPFA, Congrès, Quatrième session, 1885, p. 9-10.
} 
Ce qui est certain, c'est que ces hommes appartiennent à une certaine 'élite enseignante' qui se confond désormais avec les membres influents de la communauté française en Angleterre. Les principaux fondateurs ont des postes confortables : Jules Bué enseigne à l'université d'Oxford; Georges Petilleau est professeur de français à Charterhouse; Alfred Hamonet exerce la fonction d'examinateur de français pour le recrutement civil, militaire et médical à Londres ${ }^{27}$; Charles Cassal est professeur d'université à Londres, mais aussi examinateur ${ }^{28}$.

Les buts de cette association sont multiples; il s'agit avant tout de défendre la situation souvent difficile des enseignants, ce qui donne à la société une coloration de société à la fois «corporatiste », défendant les intérêts d'une profession, et charitable, venant en aide à des enseignants en détresse et notamment à d'anciens enseignants. La liste des adhérents fait en effet apparaître les noms d'enseignants dans des situations moins enviables: madame veuve Gaffney, femme d'un journaliste proscrit est institutrice et vit difficilement comme le montre son dossier pour la loi de réparation... mais il est vrai que Hamonet lui même se présente en 1881 comme dans la plus extrême nécessité, omettant de signaler sa véritable situation.

Ce souci de protéger la profession a constitué l'une des préoccupations constantes des enseignants français du $\mathrm{XIX}^{\mathrm{e}}$ siècle qui, par deux fois, tentent de se réunir en association, comme l'explique Paul Gerbod :

Cette présence française, à la fois concentrée dans Londres et dispersée en province, cherche, au début des années 30 , à se structurer dans le cadre d'une association nationale. [...] Cette «Society of French Teachers» [...] se propose de contribuer à fortifier l'importance et la dignité (Respectability) de la profession en admettant seulement les maîtres qui, par leurs qualités intellectuelles et morales, sont capables d'acquérir la confiance des familles (public confidence). [...] Ce n'est que dans les années 60 que cette tentative qui a dû tourner court se concrétise dans la création d'une Société des professeurs de français autour de Petilleau et d'Alphonse Mariette ${ }^{29}$.

La SNPFA bénéficie du soutien de 'personnalités' qui constituent un prestigieux comité d'honneur, présidé par Victor Hugo dont il faut rappeler qu'il avait choisi de s'installer à Jersey pour rester en terre francophone. C'est là une nouveauté qui tient au changement politique, comme le souligne Charles Cassal : la société créée en 1863 n'avait pas bénéficié d'un tel «patronage ${ }^{30}$. L'origine républicaine des membres de la société rencontre les aspirations du nouveau régime et son projet éducatif puisqu'en 1882 nous sommes au moment du vote des lois Ferry.

Au-delà de l'organisation professionnelle, la société vise à améliorer la place du français par des prises de position à la fois défensives et offensives qui s'inscrivent dans un contexte militant où il est parfois difficile de reconnaître les moyens qui visent à améliorer réellement l'apprentissage du français en Angleterre.

Le discours des sociétaires se veut alarmiste. Ces plaintes doivent bien entendu être resituées dans leur finalité. Ce discours de déploration vise à convaincre les autorités françaises de la nécessité d'aider et donc de subventionner et de promouvoir l'action de la société. Pour améliorer la situation, il faut tout d'abord consolider et préserver les positions acquises. Il faut selon eux, s'élever contre les critiques faites aux enseignants français et les retourner contre les Anglais. Car ceux-ci sont les vrais responsables. Le système anglais est jugé trop libéral : il ne pratique aucun contrôle. Tout cela renvoie plus globalement aux critiques faites à l'encontre du libéralisme anglais. Dans son

\footnotetext{
${ }^{27}$ Ibid., p. 15.

${ }^{28}$ Ibid., p. 35.

${ }^{29}$ Paul Gerbod, op. cit., p. 181-182.

${ }^{30}$ Ibid., p. 41.
} 
discours intitulé «Une Poignée de Vérités », prononcé à l'occasion du congrès de 1885 , le sociétaire Georges Petilleau fait part au public de ses observations concernant le système d'enseignement en Angleterre. Le niveau de qualification attendu des enseignants est selon lui inexistant : "en Angleterre, chose étrange, alors qu'il faut des diplômes pour être médecin, on peut s'en passer pour être maître d'école ». Il met en doute la qualité même d'établissements institués à l'économie, avec rapidité et légèreté :

Ce n'est pas difficile: on loue une semi-detached villa 'quelconque' à Balham, Fulham, Streatham ou Twickenham; on achète des meubles on the three years' system ; $[\ldots]$ on appelle cela d'un nom d'arbre 'quelconque', The Elms, the Oaks ou the Chestnuts, et voilà une École ! $!^{31}$

Hamonet s'indigne aussi :

Vous savez que le bureau de placement est le lieu où se fabrique une grande partie du personnel enseignant le français. Il suffit de se présenter dans ces officines, d'y décliner ses noms et prénoms, âge et condition, et, paraît-il, d'y faire ou souscrire un paiement quelconque pour avoir les qualités du professeur, le savoir et les aptitudes ${ }^{32}$.

Cela conduit l'ambassadeur de France, W.-H. Waddington, à déclarer :

Les Anglais sont bien en retard dans l'étude des langues modernes et surtout dans la connaissance du français,

et à s'en étonner :

c'est impardonnable chez une nation dont le bon sens commercial est légendaire ${ }^{33}$.

La Société vise aussi à structurer un milieu, à le rendre plus fécond. On juge en effet que les Français n'ont d'autre choix lorsqu'ils vivent à l'étranger que d'enseigner car l'air anglais tue leur créativité. Le Français à l'étranger n'est plus stimulé :

Les Français de Grande-Bretagne produisent fort peu d'ouvrages en dehors des livres d'enseignement qui ne sont eux-mêmes pour la plupart que des œuvres d'imitation. On dirait qu'une fois dans ce pays, nous perdons la faculté de concevoir quoi que ce soit ayant un caractère d'originalité. Quelques grands esprits l'ont habitée, je vous demande ce qu'ils y ont produit

En note, il va au devant de possibles contradicteurs :

si l'on me citait Louis Blanc je dirais que les travaux de cet historien sont les fruits de la recherche et non de l'imagination, si l'on me citait Victor Hugo je répondrai que Jersey et Guernesey sont comme il le dit lui-même, des morceaux de la Gaule cassée par la mer au huitième siècle. Je ne connais par le fait qu'Alphonse Esquiros qui ait créé un livre vraiment neuf, L’Émile du XIX ${ }^{\grave{e}}$ siècle.

Il s'agit, également, de mener un combat. La bataille de la langue est inscrite dans le thème de la Revanche. De nombreux intervenants utilisent volontiers un vocabulaire militaire : le congrès est une veillée d'armes. Les membres de la SNPFA déplorent l'intrusion d'enseignants autres que français sur le 'marché' de l'enseignement du français en Angleterre :

la profession est envahie par beaucoup de gens des deux sexes, qui, non contents d'usurper la qualité de Français, s'attribuent des aptitudes et des connaissances qu'ils n'ont pas ${ }^{34}$.

La thématique de l'envahissement est particulièrement récurrente chez Alfred Hamonet, tout comme celle de la guerre, qui découle de la première. Il stigmatise les Allemands, «race absorbante et pédante qui s'est imposée aux Anglais, il faut les empêcher » et déclare également, lors du congrès de 1882 :

\footnotetext{
${ }^{31}$ Ibid., p. 53-54.

${ }^{32}$ A. Hamonet, op. cit., p. 14.

${ }^{33}$ SNPFA, Congrès, Quatrième session, 1885, p. 19.

${ }^{34}$ A. Hamonet, op. cit., p. 15.
} 
le domaine du français est envahi, ses frontières sont renversées, une armée de Germains et de Slaves est en marche. Déjà, elle occupe plusieurs de nos postes avancés ${ }^{35}$.

Le propos d'Alfred Hamonet est nettement partisan, néanmoins, Charles Cassal plus modéré se plaint lui aussi d'une forte proportion de Suisses, de Belges, d'Anglais, d'Italiens, de Polonais, de Russes et d'Allemands parmi les 2500 personnes qui enseignent, à cette époque, le français à Londres ${ }^{36}$. Georges Petilleau qualifie ainsi la nomination du Dr Braunholtz, allemand, au poste de Lecturer in French à Cambridge d' « insulte à la fois à l'Angleterre et à la France ${ }^{37}$. A défaut d'un Français, il aurait en effet préféré que ce poste soit confié à un Anglais ${ }^{38}$.

Il faut donc lancer une véritable croisade, mais en réalité le vrai problème se situe ailleurs puisque les Français sont la cible comme les autres professeurs étrangers, d'une campagne menée notamment par le Journal of Education et son rédacteur en chef de l'époque, Francis $\mathrm{Storr}^{39}$, qui réclame l'éviction définitive des enseignants étrangers. Il s'agit donc d'organiser la résistance de la part d'enseignants français. Cette lutte contre la gallophobie est sans aucun doute également l'expression d'un sentiment communautaire qui soude les enseignants français... ${ }^{40}$.

\section{De l'enseignement à la notabilité}

La Société française est aussi conçue comme un instrument de promotion de la France à l'étranger, qui doit faire aimer la France. Les Sociétaires adoptent, en effet, pour devise : «Enseignant le français nous servons la Patrie ${ }^{41}$. Il faut ainsi contribuer à faire connaître et estimer davantage une France diminuée par la défaite ${ }^{42}$. Il s'agit aussi dans ce cadre de développer les échanges commerciaux. Les membres du comité d'honneur français expriment, en effet, l'espoir que l'action de la SNPFA puisse ouvrir «de nouvelles facilités au commerce français » ${ }^{43}$. Parallèlement à la création de la Société française des professeurs, les exilés français sont aussi à l'initiative de la création de la Chambre de commerce franco-britannique qui doit beaucoup à l'action d'un ancien professeur de Wimbledon, Tristan Duché, qui est devenu un important directeur d'usines, et dont la réussite est prolongée par celle de ses fils qui deviennent également des industriels et agrandissent à l'échelle internationale l'entreprise paternelle en ouvrant de nouvelles filiales. La création de la Chambre de commerce franco-anglaise repose largement sur Duché et ses fils ainsi que leurs amis et notamment Chevassus, proscrit installé depuis 28 ans en Grande-Bretagne. Jean Baptiste Marius Duché a épousé à Londres Marie-Hélène Chevassus. Il est viceprésident de la Chambre de commerce française de Londres en 1883 à sa création. Il en est le président en 1890, fonction qu'il abandonne en 1909. Son action est reconnue et encouragée par les autorités françaises, puisqu'il est fait chevalier de la légion d'honneur le 23 juillet 1902, officier le 23 mai 1908.

La Chambre de commerce tente, comme la Société des professeurs, de constituer en France un groupe de pression qui soutienne ses intérêts. En témoigne ce courrier adressé au ministre des Affaires étrangères :

\footnotetext{
35 Ibid., p. 5.

${ }^{36}$ SNPFA, Congrès, Quatrième session, 1885, p. 41.

${ }^{37}$ Ibid., p. 56.

${ }^{38}$ Ibid., p. 58.

39 Ibid., p. 83.

${ }^{40}$ Ibid., p. 55.

${ }^{41}$ Ibid., p. 6.

${ }^{42}$ Ibid., p. 27.

${ }^{43}$ Ibid., p. 26.
} 
Monsieur le ministre ; Londres 21 avril 1887

C'est avec une profonde satisfaction que la Chambre de commerce française de Londres apprend la formation en France d'un groupement de personnes haut placées ayant pour but un rapprochement cordial avec l'Angleterre. Un mouvement similaire existe ici présidé par Philip Stanhope, membre du Parlement, le président actuel de ces amis de la France est le major Roger Parkington ${ }^{44}$.

Enracinés en Grande Bretagne, les exilés créent donc les positions de pouvoir qui peuvent légitimer leur présence et valoriser leur action.

\section{Conclusion}

Au terme de cette étude, une évidence semble s'imposer : après l'exil, que le retour soit lié aux amnisties durant l'Empire ou à la chute de celui-ci, le proscrit qui ne vit plus hors du territoire français quitte également l'enseignement. Ceci prouverait que cette période d'inactivité politique et d'activité professionnelle contrainte sont toutes deux des parenthèses dans l'existence et la représentation de l'exil. Ceci montre à la fois la force d'un contexte mais aussi les discontinuités qu'il engendre. Y a-t-il alors une rencontre fortuite entre des hommes et une profession, des hommes et une langue ? Certainement pas. Il faut cependant travailler à l'échelle des parcours individuels pour en saisir la réalité et l'impact sur la mobilité sociale, sur les pratiques socio-culturelles, les interventions politiques mêmes. Chaque proscrit qui rentre, tente de négocier son expérience de l'étranger de façon plus ou moins visible. Ceux qui restent cherchent $a$ contrario selon leurs interlocuteurs à valoriser leur connaissance de la langue et du système éducatif ou à en minimiser les effets.

Cette recherche montre la nécessité de croiser les sources en histoire afin de prendre les acteurs au sérieux mais aussi de nuancer leurs discours. Ce croisement fécond doit aussi beaucoup à une autre rencontre, celle d'une autre discipline : l'analyse du français langue étrangère. En portant son attention sur des objets comme la production de manuels scolaires de langue qu'il considère souvent comme secondaires par rapport à une production dite culturelle, l'historien découvre tout un pan de recherches déjà amorcées et auquel la réflexion historique se doit de participer.

Qu'en est-il réellement de la place du français en milieu éducatif ? Y a-t-il démocratisation et donc développement de l'enseignement du français, voit-on au contraire son effacement? Ces questions qui intéressent plus particulièrement les chercheurs en linguistique peuvent être reposées dans notre discipline selon d'autres modalités : celle de la diffusion et de la réception de ces ouvrages, celle de la comparaison. Un vaste champ de recherche comparée s'ouvre alors sur l'expérience de l'étranger et de l'enseignement vécu par les exilés de toute l'Europe.

${ }^{44}$ Bulletin de la Chambre de commerce française de Londres, snd, 1887. 